\title{
A CARDINAL SPLINE APPROACH TO WAVELETS
}

\author{
CHARLES K. CHUI AND JIAN-ZHONG WANG
}

(Communicated by J. Marshall Ash)

\begin{abstract}
While it is well known that the $m$ th order $B$-spline $N_{m}(x)$ with integer knots generates a multiresolution analysis, $\cdots \subset V_{-1} \subset V_{0} \subset \cdots$, with the $m$ th order of approximation, we prove that $\psi(x):=L_{2 m}^{(m)}(2 x-1)$, where $L_{2 m}(x)$ denotes the $(2 m)$ th order fundamental cardinal interpolatory spline, generates the orthogonal complementary wavelet spaces $W_{k}$. Note that for $m=1$, when the $B$-spline $N_{1}(x)$ is the characteristic function of the unit interval $[0,1)$, our basic wavelet $L_{2}^{\prime}(2 x-1)$ is simply the well-known Haar wavelet. In proving that $V_{k+1}=V_{k} \oplus W_{k}$, we give the exact formulation of $N_{m}(2 x-j), j \in \mathbb{Z}$, in terms of integer translates of $N_{m}(x)$ and $\psi(x)$. This allows us to derive a wavelet decomposition algorithm without relying on orthogonality nor construction of a dual basis.
\end{abstract}

\section{INTRODUCTION}

A very natural and convenient way to introduce wavelets is to follow the notion of multiresolution analysis in $[5,7]$. Let $\phi$ be a function in $L^{2}=L^{2}(R)$ and set $\phi_{k j}(x)=\phi\left(2^{k} x-j\right)$. For each $k \in \mathbb{Z}$, denote by $V_{k}$ the $L^{2}$-closure of the algebraic span of $\left\{\phi_{k j}: j \in \mathbb{Z}\right\}$. Then the function $\phi$ is said to generate a multiresolution analysis (or approximation of $L^{2}$ ) if the following conditions are satisfied:

(i) $\cdots \subset V_{-1} \subset V_{0} \subset V_{1} \subset \cdots$;

(ii) $\operatorname{Clos}_{L^{2}}\left(\bigcup_{k \in \mathbb{Z}} V_{k}\right)=L^{2}$;

(iii) $\bigcap_{k \in \mathbb{Z}} V_{k}=\{0\}$; and

(iv) for each $k,\left\{\phi_{k j}: j \in \mathbb{Z}\right\}$ is an unconditional basis of $V_{k}$.

Suppose that $\phi$ generates a multiresolution analysis and that there exists some function $\psi$ in $L^{2}$ such that the $L^{2}$-closure $W_{0}$ of the algebraic span of $\{\psi(\cdot-j): j \in \mathbb{Z}\}$ is the orthogonal complement of $V_{0}$ in $V_{1}$. Then $\psi$ is called

Received by the editors April 30, 1990.

1980 Mathematics Subject Classification (1985 Revision). Primary 41A15, 42C05; Secondary 41A05, 41A30.

Key words and phrases. Wavelets, cardinal splines, Euler-Frobenius polynomials, wavelet decompositions, algorithms.

Supported by NSF Grants DMS-89-01345 and INT-87-12424, and SDIO/IST managed by the U.S. Army Office under Contract No. DAAL 03-90-G-0091. 
a basic wavelet relative to $\phi$. We will use the notation $V_{1}=V_{0} \oplus W_{0}$, and again set $\psi_{k j}(x)=\psi\left(2^{k} x-j\right)$ and

$$
W_{k}=\operatorname{Clos}_{L^{2}}\left(\operatorname{span}\left\{\psi_{k j}: j \in \mathbb{Z}\right\}\right) .
$$

(Note that in the wavelet literature, a normalization constant $2^{k / 2}$ is used in defining $\psi_{k j}$.) If $\psi$ is a basic wavelet relative to $\phi$, then it is clear that the "wavelet spaces" $W_{k}$, generated by $\psi$, satisfy the following properties:

(v) $V_{k+1}=V_{k} \oplus W_{k}$, all $k \in \mathbb{Z}$;

(vi) $W_{k} \perp W_{j}$, all $k \neq j$; and

(vii) $L^{2}=\bigoplus_{k \in \mathbb{Z}} W_{k}$.

As a consequence of (vii), any function in $L^{2}$ has a (unique) orthogonal decomposition in terms of functions in $W_{k}, k \in \mathbb{Z}$. This is called a (complete) wavelet decomposition. Of course, in applications, one first approximates an $f \in L^{2}$ by some $f_{k} \in V_{k}$ with a sufficiently large value of $k$ (cf. (i) and (ii)) and then obtains the (unique) wavelet decomposition of $f_{k}$, namely:

$$
f_{k}=g_{k-1}+\cdots+g_{k-\ell}+f_{k-\ell}
$$

for some sufficiently large value of $\ell$, so that $f_{k-\ell}$ has arbitrarily small $L^{2}$ norm (cf. (iii)). Here, $g_{i} \in W_{i}, i=k-\ell, \ldots, k-1$, and $f_{k-\ell} \in V_{k-\ell}$. Hence, in view of (i), (v), and (vi), the decomposition of $f_{k}$ in (1.1) is an orthogonal decomposition (in the sense that all the components $g_{k-1}, \ldots, g_{k-\ell}, f_{k-\ell}$ of $f_{k}$ are mutually orthogonal).

A standard method to determine a basic wavelet $\tilde{\psi}$ from $\phi$ can be summarized very briefly as follows: First, orthonomalize $\left\{\phi_{0 j}\right\}$, yielding $\left\{\tilde{\phi}_{0 j}\right\}$; then find the (two-scale) relation of $\tilde{\phi}$ in terms of $\left\{\tilde{\phi}_{1 j}\right\}$; and finally alternate the signs of the coefficient sequence in this two-scale relation in a clever way to form $\tilde{\psi}$ in terms of $\left\{\tilde{\phi}_{1 j}\right\}$ (cf. $\left.[5,3]\right)$. An extra feature that this basic wavelet $\tilde{\psi}$ has is that $\left\{\tilde{\psi}_{0 j}\right\}$ is an orthogonal family. For instance, if $\phi$ is the $m$ th order $B$-spline $N_{m}$, defined by convoluting the characteristic function $\chi_{(0,1)}$ with itself $m$-times (cf. [8] and [2]), then the procedure outlined above has been demonstrated for $m=4$ in [5] to yield the orthonomal (cubic spline) wavelets of Battle [1] and Lemarié [4] (also see [7]).

It is noted from these examples that even for small values of $m$ such as $m=2$ and 4 , the price to pay in order to achieve the additional orthogonality property of $\left\{\tilde{\psi}_{0 j}\right\}$ is quite high. In the first place, it imposes extra computational complexity. Indeed, even for linear and cubic splines, it is extremely difficult to obtain an explicit representation of the basic wavelet $\tilde{\psi}$ (cf. [5, 7]). Of course, for computational purposes, an expression of $\tilde{\psi}$ is not important. However, due to the orthogonality of $\left\{\tilde{\psi}_{0 j}\right\}$, the rate of decay of the sequences in the formulation for reconstructing $\tilde{\phi}_{1 j}$ in terms of $\left\{\tilde{\phi}_{0 j}\right\}$ and $\left\{\tilde{\psi}_{0 j}\right\}$, which is essential in the computational algorithms for the wavelet decomposition (1.1) (cf. $[3,6])$, seems to be somewhat slow. 
In this paper, we take a more direct approach to constructing a basic wavelet $\psi$ from $\phi$. While it is essential to generate the same wavelet spaces $W_{k}$, so that the properties (v)-(vii) are valid and the same wavelet decomposition (1.1) is attained, we do not impose orthogonality on the family $\left\{\psi_{0 j}\right\}$. Instead, we look for a $\psi$ so that the sequences for representing $\phi_{1 j}$ in terms of $\left\{\phi_{0 j}\right\}$ and $\left\{\psi_{0 j}\right\}$ have sufficiently fast rate of decay. In fact, we believe that this approach will allow us to construct a larger class of compactly supported basic wavelets $\psi$ than the class obtained by Daubechies [3]. Our results in this direction will be presented in a later paper. The objective of this paper is to demonstrate this point of view by considering the $B$-splines $N_{m}$ of arbitrary orders.

\section{MAIN RESUlts}

As usual, let $\chi$ denote the characteristic function of a set $A$. Then the $m$ th order $B$-splines $N_{m}$ are defined as follows:

$$
N_{1}(x)=\chi_{[0,1)}(x)
$$

and for $m=2,3, \ldots$,

$$
N_{m}(x)=\left(N_{m-1} * N_{1}\right)(x)=\int_{0}^{1} N_{m-1}(x-t) d t .
$$

Clearly, the support of $N_{m}$ is the interval $[0, m]$ and the $m$ th derivative of $N_{m}$ is given by

$$
N_{m}^{(m)}(x)=\sum_{j=0}^{m}(-1)^{j}\left(\begin{array}{c}
m \\
j
\end{array}\right) \delta(x-j),
$$

where $\delta$ denotes, as usual, the Dirac delta distribution with unit mass at the origin (cf. [2]). In this paper, we will consider

$$
\phi=N_{m},
$$

which generates a multiresolution analysis (i)-(iv). In fact, its order of approximation is precisely $m$. (For more details on approximation orders, see [2].)

To describe our basic wavelet $\psi$ relative to $\phi$ given by (2.2), we need the fundamental (cardinal) interpolatory spline:

$$
L_{2 m}(x)=\sum_{j \in \mathbb{Z}} \alpha_{j} N_{2 m}(x+m-j),
$$

defined uniquely by the interpolation conditions:

$$
L_{2 m}(k)=\delta_{k, 0}, \quad k \in \mathbb{Z},
$$

where $\alpha_{j}=\alpha_{j}^{(m)}$ and the standard notation for the Kronecker delta is used. That is, by considering the symbols

$$
A(z)=\sum_{j \in \mathbb{Z}} \alpha_{j} z^{j}
$$


and

$$
B(z)=\sum_{j=-m+1}^{m-1} N_{2 m}(j+m) z^{j},
$$

the fundamental interpolation conditions (2.4) can be formulated as

$$
A(z) B(z) \equiv 1 \text {. }
$$

The importance of this formulation is that the finite Laurent series $B(z)$ can be expressed as

$$
B(z)=\frac{1}{(2 m-1) !} z^{-m+1} \Pi(z),
$$

where $\Pi=\Pi_{2 m-1}$ is the well-known Euler-Frobenius polynomial of degree $2 m-2$ (cf. Schoenberg [8]). We will make use of this important information later.

Our basic wavelet $\psi=\psi_{m}$ relative to $\phi=N_{m}$ is given by

$$
\psi(x)=L_{2 m}^{(m)}(2 x-1) .
$$

Clearly, for $m=1$, when $\phi=\chi_{[0,1)}$, the corresponding basic wavelet $\psi=\psi_{1}$ is simply the Haar wavelet. In general, we must verify the properties (v)-(vii).

In the first place, $W_{0}$ is orthogonal to $V_{0}$, so that

$$
W_{k} \perp V_{k}, \quad \text { all } k \in \mathbb{Z},
$$

is trivial. Indeed, for all $\ell_{1}$ and $\ell_{2} \in \mathbb{Z}$, it follows from (2.1) and (2.4) that, with $j=\ell_{2}-\ell_{1}$,

$$
\begin{aligned}
\int_{-\infty}^{\infty} & \psi\left(x-\ell_{1}\right) \bar{\phi}\left(x-\ell_{2}\right) d x=\int_{-\infty}^{\infty} \psi(x) \phi(x-j) d x \\
& =\int_{-\infty}^{\infty} L_{2 m}^{(m)}(2 x-1) N_{m}(x-j) d x \\
& =\frac{(-1)^{m}}{2^{m}} \int_{-\infty}^{\infty} L_{2 m}(2 x-1) N_{m}^{(m)}(x-j) d x \\
& =\sum_{\nu=0}^{m} \frac{1}{2^{m}}(-1)^{m-\nu}\left(\begin{array}{c}
m \\
\nu
\end{array}\right) \int_{-\infty}^{\infty} L_{2 m}(2 x-1) \delta(x-j-\nu) d x \\
& =\sum_{\nu=0}^{m} \frac{1}{2^{m}}(-1)^{m-\nu}\left(\begin{array}{c}
m \\
\nu
\end{array}\right) L_{2 m}(2 j+2 \nu-1)=0 .
\end{aligned}
$$

On the other hand, while the inclusion $V_{0} \subset V_{1}$ is a simple consequence of the two-scale identity:

$$
\phi(x)=\sum_{j=0}^{m} 2^{-m+1}\left(\begin{array}{c}
m \\
j
\end{array}\right) \phi(2 x-j),
$$

which can be described in terms of Fourier transforms by the identity:

$$
\hat{\phi}(\omega)=\frac{1}{2} P(z) \hat{\phi}\left(\frac{\omega}{2}\right),
$$


where

$$
z:=e^{-i \frac{\omega}{2}}
$$

and

$$
P(z)=\sum_{j=0}^{m} 2^{-m+1}\left(\begin{array}{c}
m \\
j
\end{array}\right) z^{j}=2^{-m+1}(1+z)^{m} ;
$$

the inclusion $W_{0} \subset V_{1}$, so that

$$
W_{k} \subset V_{k+1}, \quad \text { all } k \in \mathbb{Z},
$$

is a consequence of the following lemma.

Lemma 1. Let $z$ be as in (2.13). Then

$$
\hat{\psi}(\omega)=\frac{1}{2} Q(z) \hat{\phi}\left(\frac{\omega}{2}\right),
$$

where

$$
Q(z):=\frac{(1-z)^{m}}{z^{m-1}} A(z) .
$$

Here, $A(z)$ is the symbol of $\left\{\alpha_{j}\right\}$ defined in (2.5). As a trivial consequence of (2.10) and (2.15), we have the required orthogonality property (vi), namely:

$$
W_{k} \perp W_{j}, \quad \text { all } k \neq j \text { in } \mathbb{Z} \text {. }
$$

So, to conclude that $\psi$ is a basic wavelet relative to $\phi$, it is sufficient to verify

$$
V_{1}=V_{0}+W_{0} \text {. }
$$

Indeed, if (2.18) holds, then (v) and (vii) follow from (2.10) and (i)-(iii). However, in order to give a computational algorithm for achieving the wavelet decomposition (1.1), we must give the explicit rule that describes the decomposition (2.18). That is, we need to determine the $\ell^{2}$ sequences $\left\{a_{n}\right\}$ and $\left\{b_{n}\right\}$ that express each $\phi_{1 \ell}, \ell \in \mathbb{Z}$, in terms of $\left\{\phi_{0 n}\right\}$ and $\left\{\psi_{0 n}\right\}$ as follows:

$$
\phi(2 x-\ell)=\sum_{n} a_{\ell-2 n} \phi(x-n)+\sum_{n} b_{\ell-2 n} \psi(x-n) \text {. }
$$

The main theorem in this paper is to determine these two sequences $\left\{a_{n}\right\}$ and $\left\{b_{n}\right\}$. To facilitate the statement of this theorem, it is more convenient to introduce two Laurent series:

$$
\begin{aligned}
G(z) & :=\frac{1}{(2 m-1) ! 2^{m}} \cdot \frac{(1+z)^{m} \Pi(z)}{z^{2 m-1}} A\left(z^{2}\right) \\
& =\frac{1}{(2 m-1) ! 2^{m}} \cdot \frac{(1+z)^{m} \Pi(z)}{z^{2 m-1}} \sum_{j \in \mathbb{Z}} \alpha_{j} z^{2 j}
\end{aligned}
$$

and

$$
\begin{aligned}
H(z) & :=\frac{-1}{[(2 m-1) !]^{2} 2^{2 m-1}} \cdot \frac{(1-z)^{m} \Pi(z) \Pi(-z)}{z^{2 m-1}} A\left(z^{2}\right) \\
& =\frac{-1}{[(2 m-1) !]^{2} 2^{2 m-1}} \cdot \frac{(1-z)^{m} \Pi(z) \Pi(-z)}{z^{2 m-1}} \sum_{j \in \mathbb{Z}} \alpha_{j} z^{2 j}
\end{aligned}
$$


Recall that $\Pi(z)$ is the Euler-Frobenius polynomial of degree $2 m-2$, with coefficients:

$$
(2 m-1) ! N_{2 m}(j), \quad j=1, \ldots, 2 m-1,
$$

(cf. (2.8) and (2.6)). We have the following result.

Theorem 1. Let $G(z)$ and $H(z)$ be defined as in (2.20) and (2.21). Then the sequences $\left\{a_{n}\right\}$ and $\left\{b_{n}\right\}$ that give the wavelet decomposition (2.19) are the coefficients of the following Laurent expansions of $G(z)$ and $H(z)$ :

$$
G(z)=\sum_{n \in \mathbb{Z}} a_{n} z^{-n}
$$

and

$$
H(z)=\sum_{n \in \mathbb{Z}} b_{n} z^{-n} .
$$

We remark that since $\Pi(z)$ is only a polynomial, it follows from (2.20)(2.23) that the rate of exponential decay of the sequences $\left\{a_{n}\right\}$ and $\left\{b_{n}\right\}$ does not exceed that of $\left\{\alpha_{[n / 2]}\right\}$. To give this rate of decay, let the roots of $\Pi=$ $\Pi_{2 m-1}$ be denoted by $r_{j}, j=1, \ldots, 2 m-2$, labeled in decreasing order; that is,

$$
r_{2 m-2}<r_{2 m-3}<\cdots<r_{m}<-1<r_{m-1}<\cdots<r_{1}<0,
$$

where $r_{1} r_{2 m-2}=\cdots=r_{m-1} r_{m}=1$ (cf. Schoenberg [8, pp. 37-38]). Then we have

$$
\alpha_{n}=O\left(\left|r_{m}\right|^{-|n|}\right), \quad n \rightarrow \pm \infty
$$

Hence, in view of the formulations (2.20)-(2.23), we have

$$
a_{n}, b_{n}=O\left(\left|r_{m}\right|^{-|n| / 2}\right), \quad n \rightarrow \pm \infty \text {. }
$$

\section{PROOFS OF RESUlts}

First, we remark that Lemma 1 can be easily verified by using Fourier transforms and the identity

$$
N_{2 m}^{(m)}(x)=\sum_{j=0}^{m}(-1)^{j}\left(\begin{array}{c}
m \\
j
\end{array}\right) N_{m}(x-j),
$$

(cf. [2]). To establish the theorem, we need the following result.

Lemma 2. Let $\Pi=\Pi_{2 m-1}$ be the Euler-Frobenius polynomials. Then

$$
\Pi(z)(1+z)^{2 m}-\Pi(-z)(1-z)^{2 m}=2^{2 m} z \Pi\left(z^{2}\right)
$$

for all $z$.

To verify this lemma, we recall the identity

$$
\frac{\Pi(z)}{(1-z)^{2 m}}=\sum_{j=0}^{\infty}(j+1)^{2 m-1} z^{j}
$$


(cf. [8]). Hence, it follows that

$$
\begin{aligned}
\frac{\Pi(z)}{(1-z)^{2 m}}-\frac{\Pi(-z)}{(1+z)^{2 m}} & =\sum_{j=0}^{\infty}(j+1)^{2 m-1}\left(z^{j}-(-1)^{j} z^{j}\right) \\
& =2^{2 m} z \sum_{k=0}^{\infty}(k+1)^{2 m-1} z^{2 k}=2^{2 m} z \frac{\Pi\left(z^{2}\right)}{\left(1-z^{2}\right)^{2 m}}
\end{aligned}
$$

which is equivalent to (3.1).

To establish Theorem 1, we first note that (2.19) is equivalent to

$$
\frac{1}{2} \hat{\phi}\left(\frac{\omega}{2}\right) e^{-i \ell \omega / 2}=\sum_{n \in \mathbb{Z}} a_{\ell-2 n} e^{-i n \omega} \hat{\phi}(\omega)+\sum_{n \in \mathbb{Z}} b_{\ell-2 n} e^{-i n \omega} \hat{\psi}(\omega)
$$

which, in view of the two-scale identities (2.12) and (2.16), is in turn equivalent to

$$
P(z) \sum_{n \in \mathbb{Z}} a_{\ell-2 n} z^{2 n-\ell}+Q(z) \sum_{n \in \mathbb{Z}} b_{\ell-2 n} z^{2 n-\ell}=1,
$$

where $P$ and $Q$ are given in (2.14) and (2.17), and the notation (2.13) is used. By a change of indices, it is clear that (3.3), and hence (2.19), is equivalent to the set of two identities:

$$
\left\{\begin{array}{l}
P(z) \frac{G(z)+G(-z)}{2}+Q(z) \frac{H(z)+H(-z)}{2}=1, \\
P(z) \frac{G(z)-G(-z)}{2}+Q(z) \frac{H(z)-H(-z)}{2}=1 .
\end{array}\right.
$$

Now, by applying Lemma 2, and recalling (2.7), (2.8), (2.20), and (2.21), it is straightforward to arrive at (3.4).

\section{DeCOMPOSITION AND RECONSTRUCTION ALGORITHMS}

The sequences $\left\{a_{n}\right\}$ and $\left\{b_{n}\right\}$ which have at least the exponential decay rate (2.25) can now be used to yield the wavelet decomposition (1.1). Indeed, writing

$$
\begin{cases}f_{j}:=\sum_{m} c_{m}^{j} \phi_{j m}, & j=k-\ell, \ldots, k, \\ g_{j}:=\sum_{m} d_{m}^{j} \psi_{j m}, & j=k-\ell, \ldots, k-1,\end{cases}
$$

we note that, as a consequence of the decomposition (2.19), the decomposition

$$
f_{j}=f_{j-1}+g_{j-1}, \quad j=k-\ell, \ldots, k,
$$

is equivalent to the recursive relations:

$$
\begin{cases}c_{\ell}^{j-1}=\sum_{n} a_{n-2 \ell} c_{n}^{j}, & j=k-\ell+1, \ldots, k, \\ d_{\ell}^{j-1}=\sum_{n} b_{n-2 \ell} c_{n}^{j}, & j=k-\ell+1, \ldots, k .\end{cases}
$$


Hence, (4.3) can be used to arrive at the wavelet decomposition (1.1), namely,

$$
f_{k}=g_{k-1}+\cdots+g_{k-\ell}+f_{k-\ell},
$$

where $f_{k-\ell} \in V_{k-\ell}$ and $g_{j} \in W_{j}, j=k-\ell, \ldots, k-1$, are formulated in (4.1).

Similarly, the two-scale relations (2.11) and (2.16) can be used to reconstruct $f_{k}$ from $f_{k-\ell}$ and $g_{k-\ell}, \ldots, g_{k-1}$. (Of course, in applications, these wavelets $g_{k-1}, \ldots, g_{k-\ell}$ have probably been processed and are necessarily different from those in (4.4).) For notational convenience, let us write

$$
P(z)=\sum_{j=0}^{m} p_{j} z^{j}
$$

(that is, $p_{j}=2^{-m+1}\left(\begin{array}{c}m \\ j\end{array}\right)$ ) and

$$
Q(z)=\sum_{j \in \mathbb{Z}} q_{j} z^{j} .
$$

Then, in view of (2.11) and (2.16), the decomposition (4.2) is equivalent to

$$
c_{\ell}^{j}=\sum_{n} p_{\ell-2 n} c_{n}^{j-1}+\sum_{n} q_{\ell-2 n} d_{n}^{j-1}, \quad j=k-\ell+1, \ldots, k .
$$

Note that $\left\{p_{n}\right\}$ is a finite sequence, and in view of (2.17) and (2.24), we have

$$
q_{n}=O\left(\left|r_{m}\right|^{-|n|}\right), \quad n \rightarrow \pm \infty .
$$

For more details on algorithms of this type and their applications, the reader is referred to Mallat [6].

\section{ACKNOWLEDGMENT}

We are very grateful to G. Battle, A. Cohen, I. Daubechies, and S. Mallat for providing us with their preprints and reprints. We would also like to thank G. Battle for giving very stimulating lectures on wavelets in the Mathematical Physics seminars at Texas A \& M University. The second author was a Visiting Scholar at the Center for Approximation Theory of Texas A \& M University during the period Sept. 1989-Dec. 1990.

\section{REFERENCES}

1. G. Battle, A block spin construction of ondelettes. Part I: Lemarié functions, Comm. Math. Phys. 110 (1987), 601-615.

2. C. K. Chui, Multivariate splines, CBMS-NSF Regional Conf. Ser. in Appl. Math. no. 54, SIAM, Philadelphia, Penn., 1988.

3. I. Daubechies, Orthonormal bases of compactly supported wavelets, Comm. Pure Appl. Math. 41 (1988), 909-996.

4. P. G. Lemarié, Ondelettes a localisation exponentielle, J. Math. Pures Appl. 67 (1988), 227-236.

5. S. G. Mallat, Multiresolution approximations and wavelet orthonormal bases of $L^{2}(R)$, Trans. Amer. Math. Soc. 315 (1989), 69-87. 
6. _ Multifrequency channel decompositions of images and wavelet models, IEEE Trans. Acoust. Speech Signal Process. 37 (1989), 2091-2110.

7. Y. Meyer, Ondelettes et functions splines, Seminaire Equations aux Derivees Partielles, École Polytechnique, Paris (Dec. 1986).

8. I. J. Schoenberg, Cardinal spline interpolation, CBMS-NSF Regional Conf. Ser. in Appl. Math., no. 12, SIAM, Philadelphia, Penn., 1973.

Department of Mathematics, Texas A \& M University, College Station, Texas 77843 E-mail address: E443cc@TAMVM1.Bitnet

Department of Mathematics, Wuhan University, Wuhan, Hubei 430072, People's RePUBLIC OF CHINA 